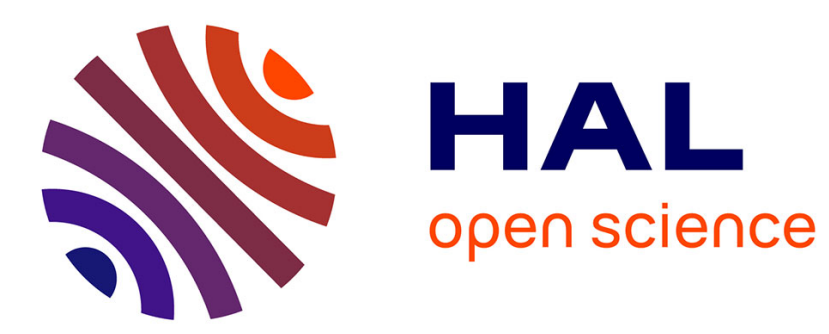

\title{
Theory of parabolic pulse propagation in nonlinear dispersion decreasing optical fiber amplifiers
}

\author{
S. Wabnitz, Christophe Finot
}

\section{To cite this version:}

S. Wabnitz, Christophe Finot. Theory of parabolic pulse propagation in nonlinear dispersion decreasing optical fiber amplifiers. Journal of the Optical Society of America B, 2008, 25 (4), pp.614-621. 10.1364/JOSAB.25.000614 . hal-00406210

\section{HAL Id: hal-00406210 https://hal.science/hal-00406210}

Submitted on 15 Apr 2010

HAL is a multi-disciplinary open access archive for the deposit and dissemination of scientific research documents, whether they are published or not. The documents may come from teaching and research institutions in France or abroad, or from public or private research centers.
L'archive ouverte pluridisciplinaire HAL, est destinée au dépôt et à la diffusion de documents scientifiques de niveau recherche, publiés ou non, émanant des établissements d'enseignement et de recherche français ou étrangers, des laboratoires publics ou privés. 


\title{
Theory of Parabolic Pulse Propagation
}

\section{in Nonlinear Dispersion Decreasing Optical Fiber Amplifiers}

\author{
Stefan Wabnitz, Christophe Finot ${ }^{*}$ \\ Institut Carnot de Bourgogne, UMR 5209 CNRS-Université de Bourgogne, 9 Av. A. Savary, \\ BP 47 870, F-21078 Dijon Cedex, FRANCE \\ *Corresponding author: christophe.finot@u-bourgogne.fr
}

We show that pulse spectral broadening in normally dispersive nonlinear fiber amplifiers may be enhanced by introducing a suitable dispersion tapering. We obtain an analytical dispersion profile which permits to reduce pulse propagation in a varying dispersion fiber to the case of an equivalent fiber with constant parameters.

OCIS codes: 190.4370, 190.5530.

\section{INTRODUCTION}

It is well-known that intense optical pulses with an initial parabolic power profile keep their shape and acquire a linear frequency chirp upon propagation in nonlinear optical fibers with normal group velocity dispersion (GVD) [1-2]. In the additional presence of linear amplification, parabolic pulses enjoy the remarkable property of representing a common asymptotic state (or attractor) for arbitrary input optical pulses with a given energy, irrespective of their initial time duration or peak power [3-7]. As well known, linear gain may be introduced in the normally 
dispersive fiber by means of external pumping, through the inherent Raman gain [7] or by doping the fiber itself with rare-earth ions $[3,8]$.

Because of their resistance to optical wave-breaking and self-similar amplification behavior, parabolic pulses or similaritons may lead to high-energy, ultrashort optical pulses when in combination with a linear dispersive grating pair [3]. Moreover, the process of similariton formation in the normal dispersion regime permits the generation of intense ultrashort pulses in fiber lasers by avoiding the pulse break-up which is typical of optical solitons in the anomalous dispersion regime [9]. In the design and optimization of similariton-based amplifiers and lasers, it is of crucial importance to evaluate the transition from a given input pulse into its asymptotic similariton solution $[8,10]$. Although this task is generally achieved by means of intensive numerical simulations, in recent work it was shown that the entire propagation dynamics of a parabolic pulse in nonlinear fiber optics amplifiers may be well described in terms of a simple finite-dimensional model involving two coupled ordinary differential equations for the adiabatic evolution of the pulse parameters [11].

Stable similariton pulses may also be generated in passive optical fibers, provided that a suitable tapering of the GVD profile (i.e., a hyperbolic decrease of dispersion with distance) is introduced [12]. First experimental demonstrations of this prediction have been reported only quite recently by using either a comb-like profiled dispersion decreasing fiber (DDF) [13], or a three-section DDF with linearly decreasing dispersion in each section [14]. A drawback of using passive DDFs is the presence of linear loss, which may negatively affect the pulse reshaping process whenever several $\mathrm{km}$ of fiber are involved. Moreover, since along a passive DDF the GVD decreases asymptotically to zero, the relative contribution of higher order GVD grows larger as the length of the DDF increases. As a result, pulse reshaping in the passive 
configuration may exhibit a strong sensitivity to third-order dispersion, that eventually lead to optical shock type instabilities [15]. It is therefore important to consider the possible benefits of combining amplification with dispersion tapering for the design of similariton-based nonlinear optical devices. Indeed, recent experiments have demonstrated that a hybrid approach for parabolic pulse generation consisting in adding Raman gain to picosecond pulse propagation in a suitably tapered DDF may permit to reduce the temporal broadening, and at the same time increase the spectral density of the generated parabolic pulses [16].

In this work we show that it is indeed possible to obtain the asymptotic evolution of pulses propagating in nonlinear dispersive fiber amplifiers with varying parameters by means of a formal description involving an equivalent amplifier with constant parameters. Indeed, we will point out that the entire optical pulse propagation in a nonlinear DDF amplifier may be exactly described whenever the dispersion tapering is obtained as the solution of a Riccati equation. Extensive numerical simulations confirm the validity of the proposed approach, and suggest that DDF fiber amplifiers may find important applications to pulse spectral broadening and supercontinuum generation, as well as telecommunication applications such as for example optical code-division multiple access.

\section{BASIC EQUATIONS}

The propagation of short optical pulses in a nonlinear optical fiber with linear gain and varying GVD may be described in terms of the dimensionless nonlinear Schrödinger equation (NLSE) with varying coefficients

$$
i \frac{\partial q}{\partial Z}-\frac{\beta}{2} D(Z) \frac{\partial^{2} q}{\partial T^{2}}+|q|^{2} q=i \delta_{0} q
$$


where the dimensionless time is $\mathrm{T}=\mathrm{t} / \mathrm{t}_{0}$, distance is $\mathrm{Z}=\mathrm{z} / \mathrm{L}_{\mathrm{NL}}=\mathrm{z} \gamma \mathrm{P}_{0}$, and $\mathrm{q}=\mathrm{Q} / \sqrt{\mathrm{P}_{0}}$ represents the dimensionless electric field, where $\mathrm{L}_{\mathrm{NL}}=1 /\left(\gamma \mathrm{P}_{0}\right)$ is the nonlinear length. Moreover, $\mathrm{t}_{0}, \mathrm{P}_{0}$ are arbitrary time and power units. In addition, $\beta=\mathrm{L}_{\mathrm{NL}} / \mathrm{L}_{\mathrm{D}}$ is the ratio between the nonlinear length and the dispersion length at $\mathrm{z}=0$, i.e. $\mathrm{L}_{\mathrm{D}}=\mathrm{t}_{0}{ }^{2} / \beta_{2}$. The function $1 \geq \mathrm{D}(\mathrm{z})>0$ describes the longitudinal variation of the fiber GVD along its length. Finally, $\delta_{0}=\Gamma_{0} / 2=(\mathrm{G}-\alpha) \mathrm{L}_{\mathrm{NL}} / 2, \mathrm{G}\left[\mathrm{m}^{-1}\right]$ and $\alpha\left[\mathrm{m}^{-1}\right]$ are the power gain and loss coefficients of the fiber. It is convenient to rewrite Eq.(1) in terms of the new distance coordinate $\xi$ and field amplitude $u$ defined as [12]

$$
\begin{aligned}
& \xi \equiv \int_{0}^{Z} D(X) d X \\
& u=\frac{q}{\sqrt{D(Z)}}
\end{aligned}
$$

One obtains

$$
\frac{\partial u}{\partial \xi}=\frac{\partial}{\partial Z}\left(\frac{q(Z, T)}{\sqrt{D(Z)}}\right) \frac{d Z}{d \xi}=\frac{\partial q}{\partial Z} \frac{1}{D^{3 / 2}}-\frac{1}{2} \frac{q}{\sqrt{D}} \frac{1}{D^{2}} \frac{d D}{d Z}
$$

When one divides each term in Eq.(1) by $\mathrm{D}^{3 / 2}$, taking into account Eqs.(2-3) one finally obtains the NLSE with constant coefficients

$$
i \frac{\partial u}{\partial \xi}-\frac{\beta}{2} \frac{\partial^{2} u}{\partial T^{2}}+|u|^{2} u=i \delta u
$$

where we defined the virtual constant (i.e., $\xi$-independent) gain coefficient $\delta=\Gamma / 2$ and

$$
\Gamma=\frac{1}{D(Z)}\left(\Gamma_{0}-\frac{1}{D(Z)} \frac{d D(Z)}{d Z}\right)
$$


Since for a DDF one has $\mathrm{dD} / \mathrm{dZ}<0$, Eqs.(4-5) mean that, in a properly designed DDF, the evolution of the re-normalized complex amplitude u obeys the NLSE with both constant GVD and constant virtual linear gain coefficient $\delta$. Eq.(4) shows that the constant GVD is equal to the dispersion at the input end of the DDF (i.e., where $\mathrm{D}(\mathrm{Z}=0)=1$ ), whereas Eq.(5) reveals that the constant gain coefficient $\delta$ (or $\Gamma$ ) is obtained by locally adding two Z-varying contributions, namely the sum of the physical gain coefficient $\delta_{0}$ and of the virtual gain which is induced by the dispersion profile, both divided by the dispersion decrease function $D(Z)$. As a result, we anticipate that dispersion tailoring may permit to reduce, with respect to a constant dispersion fiber (CDF), the linear amplifier gain which is necessary to achieve a certain amount of spectral broadening of the input pulses. Note however that the DDF length say, $\mathrm{L}_{\mathrm{DDF}}$, in the presence of a given physical gain coefficient $\delta_{0}$, should be increased with respect to the length $L$ of a CDF with the corresponding virtual gain coefficient $\delta$, according to the relationship

$$
\int_{0}^{\mathrm{L}_{\mathrm{DDF}}} \mathrm{D}(\mathrm{Z}) \mathrm{dZ}=\mathrm{L}
$$

From Eq.(5), for any given set of $\delta$ and $\delta_{0}$, the optimal dispersion profile $\mathrm{D}(\mathrm{Z})$ of the DDF is obtained as the solution of the first-order nonlinear Riccati differential equation

$$
\frac{d D}{d Z}=\Gamma_{0} D-\Gamma D^{2}, \quad D(Z=0)=1
$$

The solution of the above equation is well known in the theory of ordinary differential equations. By following the simple steps as outlined in the appendix, one obtains from Eq.(7) the dispersion profile

$$
D(Z)=\frac{\Gamma_{0}}{\Gamma}\left\{1+\frac{\Gamma-\Gamma_{0}}{\Gamma_{0}+\Gamma\left(e^{\Gamma_{0} Z}-1\right)}\right\},
$$


which permits to calculate the integral in Eq.(6) as

$$
L=\int_{0}^{L_{D D F}} D(Z) d Z=\frac{1}{\Gamma}\left[\log \left\{\Gamma\left(e^{\Gamma_{0} L_{D D F}}-1\right)+\Gamma_{0}\right\}-\log \Gamma_{0}\right]
$$

so that

$$
L_{D D F}=\frac{1}{\Gamma_{0}}\left[\log \left\{\Gamma_{0}\left(e^{\Gamma L}-1\right)+\Gamma\right\}-\log \Gamma\right] .
$$

\section{ANALYSIS}

We may express the field $u$ in Eq.(4) in terms of its intensity $\rho$ and phase $\phi$, or instantaneous frequency $\omega(\mathrm{T})=-\partial \phi / \partial \mathrm{T}$

$$
\mathrm{u}(\mathrm{T}, \boldsymbol{\xi})=\sqrt{\rho(\mathrm{T}, \xi)} \exp [\mathrm{i} \phi]=\sqrt{\rho(\mathrm{T}, \xi)} \exp \left[-\mathrm{i} \int^{\mathrm{T}} \omega\left(\mathrm{T}^{\prime}, \xi\right) \mathrm{dT}^{\prime}\right]
$$

We shall consider the propagation of initially chirp-free parabolic pulses of the type

$$
\left\{\begin{array}{l}
\rho(\mathrm{T}, \xi)=\rho_{0}(\xi)\left(1-\frac{\mathrm{T}^{2}}{\mathrm{~T}_{0}^{2}(\xi)}\right) \\
\omega(\mathrm{T}, \xi)=\mathrm{C}_{0}(\xi) \mathrm{T}
\end{array}\right.
$$

for $|\mathrm{T}|<\mathrm{T}_{0}(\xi) \quad\left(\rho=\omega=0\right.$ otherwise), with $\mathrm{C}_{0}(\xi=0)=0$, in a DDF with initial (i.e., at $\left.\xi=0\right)$ normal dispersion at $\lambda_{0}=1550 \mathrm{~nm}$ of $\mathrm{D}_{0}=-5 \mathrm{ps} /(\mathrm{nm} \mathrm{km})$. Note that the root-mean-square time width $\mathrm{T}_{\mathrm{rms}}$ and the full-width-at-half-maximum $\mathrm{T}_{\mathrm{fwhm}}$ of the pulse (11-12) are related as follows: $T_{r m s}=2 T_{0} / \sqrt{5}=\sqrt{2 / 5} T_{f w h m}$. The asymptotic amplification regime is analytically described by Eqs. (11-12) with [2-5] 


$$
\begin{aligned}
& \rho_{0}^{S}=\sqrt[3]{U_{0}^{2} \delta^{2}} \exp [4 \delta \xi / 3] /(2 \sqrt[3]{\beta}) \equiv \rho_{0}^{S S} \exp [4 \delta \xi / 3] \\
& T_{0}^{S}=3 \sqrt[3]{U_{0} \beta / \delta^{2}} \exp [2 \delta \xi / 3] / 2 \equiv T_{0}^{S S} \exp [2 \delta \xi / 3] \\
& C_{0}^{S}=2 \delta / 3 \beta \equiv C_{0}^{S S}
\end{aligned}
$$

where the input pulse energy $\mathrm{U}_{0}=4 \rho_{0}(\xi=0) \mathrm{T}_{0}(\xi=0) / 3$.

To be specific, let us consider the following typical fiber parameters: the effective area is $\mathrm{A}_{\text {eff }}=40 \mu \mathrm{m}^{2}$, the nonlinear index is $\mathrm{n}_{2}=3.2 \times 10^{-20} \mathrm{~m}^{2} / \mathrm{W}$, and the SPM coefficient is $\gamma=3.2$ (W $\mathrm{km})^{-1}$. Moreover, we set $\mathrm{t}_{0}=1$ ps and $\mathrm{P}_{0}=10 \mathrm{~W}$, so that $\mathrm{L}_{\mathrm{NL}}=31 \mathrm{~m}$ and $\beta=0.197$. Figure 1 (a) shows the solution (8) for the dispersion profile $D(Z)$ corresponding to the virtual gain coefficient $\delta=0.5$, and to different values of the physical linear gain coefficient $0 \leq \delta_{0} \leq \delta$. As it can be seen, in each case the fiber dispersion reduction coefficient $D(Z)$ rapidly decreases in the first $100 \mathrm{~m}$ of $\mathrm{DDF}$, whereas $\mathrm{D}(\mathrm{Z})$ decreases only slowly with $\mathrm{Z}$ at longer lengths, in particular for large values of the physical gain $\delta_{0}$.

Figure 1(b) shows the dispersion profile $\mathrm{D}(\mathrm{Z})$ of Eq.(8) corresponding to the real DDF gain coefficient $\delta_{0}=0.05$ (this means a physical gain of $14 \mathrm{~dB} / \mathrm{km}$ for the choice of fiber parameters as given above), and to different values of the virtual gain coefficient $\delta$ (namely, $\delta=0.125,0.25$ and 1, respectively) of the equivalent CDF (see Eq.(4)). As it can be seen, the rate of decrease of the fiber dispersion coefficient $D(Z)$ grows larger with $\delta$. In particular, for $\delta / \delta_{0}=20$ the dispersion decrease is mostly concentrated in the first $10 \%$ of $\mathrm{L}_{\mathrm{DDF}}$. Moreover, in this case in most of the DDF the dispersion is close to its asymptotic value $\mathrm{D}(\mathrm{Z} \rightarrow \infty)=0.05$ (so that the actual DDF dispersion is equal to $-0.25 \mathrm{ps} /(\mathrm{nm} . \mathrm{km})$ ). This fact has two consequences: first, as the GVD is mostly close to its zero value, it is likely that the pulse amplification will be strongly affected by third-order dispersion. Second, it should be expected that pulse amplification in the DDF is not 
much different from the amplification that would be observed in a CDF with the dispersion value equal to the average (over the length $\mathrm{L}_{\mathrm{DDF}}$ ) dispersion of the DDF, which obviously reduces the interest in obtaining a special dispersion profiling as in Eq.(8). Therefore, it appears that it is preferable to introduce a dispersion profile $\mathrm{D}(\mathrm{Z})$ associated with a smaller virtual CDF gain coefficient such as $\delta=0.25$ or $\delta=0.125$ (see Fig. 1(b)), since in these cases the DDF dispersion remains mostly above the corresponding asymptotic values of $-1 \mathrm{ps} /(\mathrm{nm} \mathrm{km})$ or $-2 \mathrm{ps} /(\mathrm{nm} \mathrm{km})$, respectively.

In addition to the dispersion profile information that is provided by Fig. 1, it is important to evaluate the real DDF length that in each case corresponds to a given length of the virtual CDF as described by Eq.(4). This information is obtained by Eq.(10) and it is displayed in Fig. 2, which illustrates in real units the DDF length as a function of the physical linear gain $\delta_{0}$, for a given value of the virtual gain coefficient $\delta=0.5$. In Fig. 2(a) the length of the virtual CDF was set equal to $150 \mathrm{~m}$ : with this choice, the physical DDF gain of $14 \mathrm{~dB} / \mathrm{km}$ (which corresponds to $\delta_{0}=0.05$ ) leads to a DDF length of $1 \mathrm{~km}$ (see vertical arrow in Fig. 2(a)). Whereas in Fig. 2(a) the horizontal arrow indicates the DDF length in the limit case of a passive (i.e., $\delta_{0} \rightarrow 0$ ) DDF. As it can be seen in Fig. 2(a), with $\delta_{0}<0.1$ the necessary DDF length rapidly grows up to large values. Fig. 2(b) was obtained for $\delta=0.25$ : in this case the effective CDF length was increased from 150 to $350 \mathrm{~m}$, so that again the DDF gain of $14 \mathrm{~dB} / \mathrm{km}$ (i.e., $\delta_{0}=0.05$ ) results in a $1 \mathrm{~km}$ long DDF (see arrow in Fig. 2(b)).

As we intend to exploit the active DDF for pulse reshaping applications, an important question is which is the minimum DDF length that is necessary for entering into the so-called similariton propagation regime [8, 10]. Indeed, by applying to our case the analysis of ref.[11], 
we may confirm that a $1 \mathrm{~km}$ long DDF with $\delta_{0}=0.05$, and a CDF length of either 150 or $350 \mathrm{~m}$ (for $\delta=0.5$ or 0.25 , respectively) is a sufficient length for reaching the self-similar or nonlinear amplification regime. For example, Fig. 3 was obtained (as figure 4 (a-b) of ref.[11]) from the finite-dimensional description of the evolution of the parabolic pulse parameters along the CDF: the empty dots indicate the distance for entering into the self-similar or similariton amplification regime. Fig. 3 corresponds to an input a parabolic pulse as in Eqs.(11-12) with the energy $\mathrm{U}_{0}=94 \mathrm{pJ}$, which propagates as described by the equivalent CDF equation (4) with $\beta=0.2$ and $\delta=0.25$. As it can be seen, the shortest distance is obtained in correspondence of an input RMS pulse width $\mathrm{T}_{\mathrm{rms}}^{\mathrm{in}}=\mathrm{T}_{\mathrm{rms}}^{\mathrm{SS}}=2 \mathrm{~T}_{0}^{\mathrm{SS}} / \sqrt{5}$, where $\mathrm{T}_{0}^{\mathrm{SS}}$ is defined by the second of equations (13). The plot of Fig. 3 confirms that the CDF length of $350 \mathrm{~m}$ is the minimum distance that is required in order that the amplification of the parabolic pulse fully enters into the self-similar amplification regime.

A parameter that permits evaluate the improvement which is brought about by introducing a dispersion decreasing profile is the ratio between the products of the physical gain coefficient times the length of the DDF, and of the same parameters for the virtual CDF, or $\mathrm{R}=\mathrm{L}_{\mathrm{DDF}} \delta_{0} /(\mathrm{L} \delta)$. Figure 4 shows the dependence of the DDF gain/length product decrease factor $\mathrm{R}$ as a function of the physical gain coefficient $\delta_{0}$ for a CDF length of $350 \mathrm{~m}$ and for the virtual gain $\delta=0.25$ : the arrow corresponds to a $1 \mathrm{~km}$ long DDF as in figure 2(b). Figure 4 shows that a $1 \mathrm{~km}$ long DDF with the physical gain coefficient $\delta_{0}=0.05$ leads to about $30 \%$ reduction of the gain/length product with respect to the equivalent virtual CDF. This means that, for relatively small values of the physical gain coefficient, the virtual gain improvement which is introduced by dispersion tapering overcompensates the drawback of the length increase of the DDF with respect to the 
equivalent CDF. This result is due to the nonlinear nature of the Riccati differential equation (7) which determines the dispersion profile $\mathrm{D}(\mathrm{Z})$.

It is interesting to consider the case of a DDF with an initially (i.e., at $\mathrm{z}=0$ ) twice larger (in absolute value) normal dispersion, i.e., we set $\mathrm{D}_{0}=-10 \mathrm{ps} /(\mathrm{nm} \mathrm{km})$. With the choice $\mathrm{t}_{0}=1 \mathrm{ps}$ and $\mathrm{P}_{0}=20 \mathrm{~W}$, one obtains $\mathrm{L}_{\mathrm{NL}}=16 \mathrm{~m}$; note that the dimensionless $\beta$ parameter remains unchanged at $\beta=0.197$. In Fig. 5(a) we compare the DDF dispersion profile that was previously shown in Fig. 1(b) (for $\delta_{0}=0.05, \delta=0.25$ with $\mathrm{L}_{\mathrm{NL}}=31 \mathrm{~m}$ ), with the dispersion profile that is obtained with $\mathrm{L}_{\mathrm{NL}}=16 \mathrm{~m}$ and $\delta_{0}=0.025$ (i.e., still $14 \mathrm{~dB} / \mathrm{km}$ of physical gain for the DDF in real units, and $\beta=0.197$ whenever the initial dispersion is $\left.\mathrm{D}_{0}=-10 \mathrm{ps} /(\mathrm{nm} \mathrm{km})\right)$. The virtual CDF gain coefficient is maintained unchanged at $\delta=0.25$ in both cases. In Fig. 5(b) we show, as in Fig. 2, the DDF length as a function of its gain coefficient $\delta_{0}$, in the case of the nonlinear distance $\mathrm{L}_{\mathrm{NL}}=16 \mathrm{~m}$. Here the virtual gain coefficient of the associated CDF is $\delta=0.25$, and its length is $175 \mathrm{~m}$. The arrow indicates the case of a gain equal to $14 \mathrm{~dB} / \mathrm{km}$ for the DDF (that is, $\delta_{0}=0.025$ ) and shows that $\mathrm{L}_{\mathrm{DDF}}$ is again equal to about $1 \mathrm{~km}$. By computing a plot similar to Fig. 3, the choice of a 175 m length for the CDF corresponds to the minimum distance for entering into the self-similar regime for a parabolic pulse with input pulse energy $\mathrm{U}_{0}=190 \mathrm{pJ}$ that propagates in a CDF with the virtual gain coefficient $\delta=0.25$ and $\mathrm{L}_{\mathrm{NL}}=16 \mathrm{~m}$.

\section{PULSE EVOLUTION}

In this section we shall investigate in some details the pulse reshaping action of an active DDF with a dispersion decreasing profile adapted to the physical distributed gain as given by the solution (8) of section 2. In Fig. 6 we present a comparison of the evolution with distance of the 
RMS time width of different, but initially chirp-free, pulses in a CDF (i.e., with $D(Z)=1$ ) and in a DDF. In both cases the physical gain coefficient was set to $\delta_{0}=0.1$, which corresponds to $28 \mathrm{~dB}$ over the $1 \mathrm{~km}$ length of the fiber amplifier with the parameters as defined in Section 3 and the initial normal dispersion at $\lambda_{0}=1550 \mathrm{~nm}$ of $\mathrm{D}_{0}=-5 \mathrm{ps} /(\mathrm{nm} \mathrm{km})$, and a virtual gain coefficient $\delta=0.5$. We deliberately used here a linear gain which is twice as large as the value of $14 \mathrm{~dB} / \mathrm{km}$ that was used in the previous section, in order to clearly show the convergence into the selfsimilar parabolic pulse (11-13) of different input pulses, for distances well within the DDF length of $1 \mathrm{~km}$. In Fig. 6, the evolution in the DDF or the CDF of the RMS time width of a Gaussian pulse with the initial FWHM duration of 10 ps (hence 8.5 ps of RMS duration, since for a Gaussian pulse $T_{f w h m}=1.1774 T_{r m s}$ ) and $10 \mathrm{~W}$ peak power is indicated by a dot-dashed and a dotted curve, respectively. Whereas the corresponding RMS time width of an initial parabolic pulse with the same 10 ps FWHM (hence 6.3 ps RMS) time duration and peak power is indicated by a solid and a dashed curve, respectively. As it can be seen in Fig. 6, after a certain distance (about $400 \mathrm{~m}$ in the $\mathrm{CDF}$ and $700 \mathrm{n}$ in the DDF) the two different input pulses converge to the same self-similar parabolic attractor. Moreover, in the similariton regime the time width of pulses in the DDF is considerably shorter than in the CDF: for example, at $1 \mathrm{~km}$ the output pulse RMS duration is equal to about 35 ps for the DDF versus 70 ps for the CDF. On the other hand, the evolution of the spectral width of the different input pulses in the two fibers as shown by Fig. 7 shows that the DDF leads to much larger spectral broadening than the CDF. Indeed, the output spectral width of the pulses they emerge from the $1 \mathrm{~km}$ long DDF is equal to $11.1 \mathrm{THz}$, as opposed to $3.7 \mathrm{THz}$ for the CDF.

The details of the intensity profiles in both the time and the frequency domains of the Gaussian and the parabolic pulses as they emerge from the either the CDF or the DDF are shown 
in the top and bottom part of Fig. 8, respectively. As it can be seen in Fig. 8, the peak power of pulses from the DDF is nearly doubled with respect to the CDF, whereas the temporal width is halved. At the same time, Fig. 8 confirms that the spectral broadening of both parabolic and Gaussian pulses in the DDF is increased by about a factor of three with respect to the CDF. It is interesting to point out that, following the analysis of Sections 2-3, the same pulse evolution that is observed in the DDF of Figs. 6-8 could be observed in an equivalent CDF with a linear gain coefficient increased by a factor of five, that is as high as $140 \mathrm{~dB} / \mathrm{km}$ (i.e., $\delta_{0}=0.5$ in dimensionless units). However, following Eq.(9), the length of this CDF could be reduced to just $250 \mathrm{~m}$, so that the actual integrated gain of the equivalent CDF would need to be equal to $35 \mathrm{~dB}$. These considerations are confirmed by the numerical solution of Eq.(4) with the parabolic pulse input condition (11-12), as shown by the plots of Fig. 9.

The main advantage of describing the propagation in the DDF in terms its equivalent virtual CDF, is that this permits using the known asymptotic results of Eq.(13) for the analytical prediction of the evolution of the parabolic pulse in the dispersion tapered amplifier. For example, by using Eqs. (11-13) one obtains (see Fig. 10(a)) the dependence of the optimal input FWHM time width $\mathrm{T}_{\text {fwhm }}^{\mathrm{in}}=\mathrm{T}_{\mathrm{rms}}^{\mathrm{in}} \sqrt{5 / 2}$ of a parabolic pulse as a function of the input pulse energy, for different virtual gain coefficients $\delta=0.25$ or $\delta=0.125$ of the equivalent $C D F$, and the fixed value of $\delta_{0}=0.05$ for the physical gain coefficient. As it can be seen from figure 10(a), for input pulse energies $\mathrm{U}_{0}$ close to $100 \mathrm{pJ}$, the input FWHM time width decreases from 10 ps to 6 ps as the CDF virtual gain coefficient (and the corresponding dispersion profile $\mathrm{D}(\mathrm{Z})$ of the $\mathrm{DDF}$ ) is varied from $\delta=0.125$ up to $\delta=0.25$.

In a similar manner, one may easily estimate the output FWHM spectral width of the linearly chirped parabolic pulses as they emerge from a given length of DDF (say, $1 \mathrm{~km}$ ), by calculating 
the spectral width of the pulse at the output of the corresponding virtual CDF whose length is given by Eq.(9). Indeed, in the self-similar or similariton regime the FWHM spectral width is $\mathrm{F}_{\text {fwhm }}^{\text {out }}=\mathrm{F}_{\mathrm{rms}}^{\text {out }} \sqrt{5 / 2}$, where $\mathrm{F}_{\mathrm{rms}}^{\text {out }}=\mathrm{T}_{0}^{\mathrm{S}} \mathrm{C}_{0}^{\mathrm{S}} / 2 \pi$. Figure 10 (b) illustrates the prediction of Eqs. (1113) for the output FWHM spectral width of a parabolic pulse as a function of the input pulse energy, for a fixed DDF length of $1 \mathrm{~km}$, the physical gain coefficient $\delta_{0}=0.05$, and different virtual gain coefficients $\delta$ of the associated equivalent CDF (corresponding to the different dispersion profiles which are shown in Fig. 1). As it can be seen from Fig. 10(b), for input pulse energies $\mathrm{U}_{0}$ close to $100 \mathrm{pJ}$, the output FWHM spectral width increases from $2.8 \mathrm{THz}$ up to 4.5 $\mathrm{THz}$ as the CDF virtual gain coefficient (and the corresponding dispersion profile $\mathrm{D}(\mathrm{Z})$ of the DDF) is varied from $\delta=0.125$ up to $\delta=0.25$.

As shown in Fig. 11(a), we have also applied the exact asymptotic expressions (13) to calculate the optimal input FWHM time width of a parabolic pulse versus the input pulse energy for the dispersion profiles shown in Fig. 5. Here we kept the DDF length fixed to $1 \mathrm{~km}$, whereas its physical gain coefficient is $\delta_{0}=0.025$, and the virtual gain coefficient of the associated CDF is $\delta=0.25$. Finally, Fig. 11(b) shows the corresponding output FWHM spectral width of the parabolic pulse as a function of the input pulse energy, for the same choice of DDF and CDF parameters.

\section{EFFECTS OF THIRD-ORDER DISPERSION}

As mentioned in the introduction, one of the major obstacles to the use of a passive DDF for similariton generation is the presence of third-order dispersion (TOD) which leads to propagation instabilities or shock formation [15,17]. Let us briefly consider the influence of TOD on 
parabolic pulse propagation in a DDF amplifier: the evolution of the pulse field envelope $\mathrm{Q}$ is described by the extended amplified NLSE

$$
i Q_{z}+\frac{\beta_{2}(z)}{2} \frac{\partial^{2} Q}{\partial T^{2}}-i \frac{\beta_{3}}{6} \frac{\partial^{3} Q}{\partial T^{3}}+\gamma|Q|^{2} Q=i \delta_{0} Q
$$

where $\beta_{3}$ is the third-order dispersion. Figures $12(a-b)$ show the input (dashed grey curve) and output (after $1 \mathrm{~km}$ DDF with the physical gain $\delta=0.1$ ) profile (in time and frequency domains, respectively) of an initial $10 \mathrm{~W}$ peak power, $10 \mathrm{ps}$ FWHM parabolic pulse as it emerges from a DDF with no TOD (solid curves), and with all other parameters as in the case of Fig. 8. This should be compared with Figs. 12(c-d), where in numerically solving Eq.(14) the TOD was increased from zero up to $\beta_{3}=0.025 \mathrm{ps}^{3} / \mathrm{km}$ : as it can be seen, at $755 \mathrm{~m}$ the pulse exhibits a significant temporal and spectral asymmetry. Next, Figs. 13(a-b) show that after $779 \mathrm{~m}$ of DDF the pulse develops a shock in its leading edge, which corresponds in the spectral domain to significant amount of red-shifted radiation. Additionally, the plots of Figs. 13(c-d) show that when the TOD is reduced down to $\beta_{3}=0.015 \mathrm{ps}^{3} / \mathrm{km}$, the shock only develops after a $974 \mathrm{~m}$ long DDF. An approximate but analytical prediction for the critical distance at which a TOD-induced shock may be observed in an active DDF may be obtained from the Kodama's inequality [17]

$$
\left|\frac{\beta_{3}}{\sqrt[3]{-\beta_{2}}}\right|>\frac{1}{4} \sqrt{\frac{L_{N L}}{\rho_{0}}}
$$

where $\rho_{0}$ is the dimensionless peak power of the pulse. Eq.(15), which was rigorously obtained for square optical pulses (i.e., nonreturn-to-zero or NRZ pulses), predict that a shock wave type of instability develops on the pulse whenever the TOD is large enough that the inequality is satisfied. It turns out that one may predict (with about $10 \%$ accuracy) the onset of an optical shock as the peak power of the pulse reaches $450 \mathrm{~W}$ at $779 \mathrm{~m}$ (see Fig. 13(a)), or $900 \mathrm{~W}$ at 
$974 \mathrm{~m}$ (see Fig. 13(b)), provided the path-average values of the GVD $\beta_{2}$ and of the pulse peak power $\rho_{0}$ are used in Eq.(15). The advantage of using an active DDF in order to reduce the impact of TOD is clear from both the left-hand side of Eq.(15), and the nonzero asymptotic value $\underline{D}(Z>>1)=\Gamma_{0} 0$ of the dispersion profile (8). As shown by Fig. 1(a), in contrast with the passive case, in the active DDF the second-order dispersion does not vanish, hence the generated parabolic pulse will be less impacted by TOD. Note finally that, although in solving Eq.(14) we considered a TOD that is constant with $\mathrm{z}$, we may anticipate that a suitable TOD management may permit to counteract its negative influence on pulse propagation in DDF amplifiers. However the detailed analysis of the influence of TOD on pulse reshaping by means of DDF amplifiers is beyond the scope of this paper, and we plan to discuss this issue elsewhere.

\section{CONCLUSIONS AND ACKNOWLEDGEMENTS}

In this work we presented a theoretical analysis of pulse propagation in a varyingdispersion, nonlinear optical fiber amplifier. We pointed out an analytical dispersion profile which permits to reduce optical pulse propagation to a description in terms of the amplified NLSE with constant parameters. We have analysed the influence of both the physical gain and of the dispersion tapering (or virtual gain coefficient) on the pulse reshaping properties of the active DDF. In particular, our analysis permits to obtain analytic relationships for both the optimal input pulse width and the corresponding output spectral width of the optical pulses. Additionally, by exploiting the known properties of the equivalent constant parameters amplified NLSE, one may determine, hence control the minimum DDF length which leads to the asymptotic amplification regime. Let us note that the proposed guidelines for the dispersion profile may be quite easily adapted to a comb like fiber set-up, where a set of carefully chosen normally 
dispersive fibers mimics the continuous dispersion decrease. An added benefit of using an active DDF, with respect to the passive case, is that in the active case the optimal dispersion profiles do not vanish as the DDF length grows larger, which reduces the deleterious influence of higher order dispersion. Finally, we provided a simple method to estimate the threshold for the onset of TOD-driven pulse instabilities in active DDFs.

We are grateful to A. Sysoliatin for several discussions on the practical design of dispersion decreasing optical fibers. This research was supported by the Agence Nationale de la Recherche (SUPERCODE project) and by the Conseil Régional de Bourgogne.

\section{APPENDIX}

In order to solve Eq.(7) all that is needed is a particular solution. Take for example the constant solution

$$
\bar{D}=\frac{\Gamma_{0}}{\Gamma}
$$

Then the new function $d(Z)$ is defined as

$$
d(Z)=\frac{1}{D(Z)-\bar{D}}
$$

obeys the simple linear ordinary differential equation

$$
d^{\prime}=\Gamma_{0} d+\Gamma
$$

whose general solution is

$$
d(Z)=C e^{\Gamma_{0} Z}-\frac{\Gamma}{\Gamma_{0}}
$$


The integration constant $C$ is determined by the condition that $D(Z=0)=1$, so that one finally obtains the required dispersion profile as given by Eq.(8). 


\section{Reference List}

1. D. Anderson, M. Desaix, M. Karlsson, M. Lisak, and M.L. Quiroga-Teixeiro, "Wavebreaking-free pulses in nonlinear-optical fibers", J. Opt. Soc. of Am. 10, 1185-1190 (1993).

2. J. M. Dudley, C. Finot, G. Millot, and D. J. Richardson, "Self-similarity and scaling phenomena in nonlinear ultrafast optics," Nat. Phys. 3, 597-603 (2007).

3. M.E. Fernmann, V.I. Kruglov, B.C. Thomsen, J.M. Dudley, J.D. Harvey, "Self-similar propagation and amplification of parabolic pulses in optical fibers", Phys. Rev. Lett. 84, 6010-6013 (2000).

4. V.I. Kruglov, A.C. Peacock, J.M. Dudley, and J.D. Harvey, "Self-similar propagation of high power parabolic pulses in optical fiber amplifiers", Opt. Lett. 25, 1753-1755 (2000).

5. V.I. Kruglov, A.C. Peacock, J.D. Harvey, J.M. Dudley, "Self-similar propagation of parabolic pulses in normal dispersion fiber amplifiers", J. Opt. Soc. Am. B 19, 461-469 (2002).

6. C. Finot, G. Millot, and J.M. Dudley, "Asymptotic characteristics of parabolic similariton pulses in optical fiber amplifiers", Opt. Lett. 29, 2533-2535 (2004).

7. C. Finot, G. Millot, S. Pitois, C. Billet, J.M. Dudley, "Numerical and experimental study of parabolic pulses generated via Raman amplification in standard optical fibers", IEEE J. Sel. Top. Quantum Electron. 10, 1211-1218 (2004).

8. C. Billet, C.M. Dudley, N. Joly, J.C. Knight, "Intermediate asymptotic evolution and photonic bandgap fiber compression of optical similaritons around $1550 \mathrm{~nm}$ ”, Opt. Express 13, 3236-3241 (2005).

9. O. Ilday, J.R. Buckley, W.J. Clark, F.W. Wise, "Self-similar evolution of parabolic pulses in a laser”, Phys. Rev. Lett. 92, 2319021-4 (2004). 
10. C. Finot, F. Parmigiani, P. Petropoulos, D.J. Richardson, "Parabolic pulse evolution in normally dispersive fiber amplifiers preceding the similariton formation regime“, Opt. Express 14, 3161-3170 (2006).

11. S. Wabnitz, "Analytical dynamics of parabolic pulses in nonlinear optical fiber amplifiers", Photonics Techn. Lett. 19, 507-509 (2007).

12. T. Hirooka, and M. Nakazawa, "Parabolic pulse generation by use of a dispersion-decreasing fiber with normal group velocity dispersion”, Opt. Lett. 29, 498-500 (2004).

13. B. Kibler, C. Billet, P.A. Lacourt, R. Ferriere, L. Larger, and J.M. Dudley, "Parabolic pulse generation in comb-like profiled dispersion decreasing fibre", Electron. Lett. 42, 965-966 (2006)

14. A. Plocky, A. A. Sysoliatin, A. I. Latkin, V. F. Khopin, P. Harper, J. Harrison, and S. K. Turitsyn, "Experiments on the generation of parabolic pulses in waveguides with lengthvarying normal chromatic dispersion," JETP Letters 85, 319-322 (2007).

15. A. Latkin, S. Turitsyn, and A. Sysoliatin, "Theory of parabolic pulse generation in tapered fiber", Opt. Lett. 32, 331-333 (2007)

16. C. Finot, B. Barviau, G. Millot, A. Guryanov, A. Sysoliatin, and S. Wabnitz, "Parabolic pulse generation with active or passive dispersion decreasing optical fibers", Optics Express 15, $15824-15835$ (2007).

17. Y. Kodama, "The Whitham equations for optical communications: mathematical theory of NRZ”, SIAM J. Appl. Math. 59, 2162-2192 (1999). 


\section{Figure Captions}

1. Fiber length dependence of the dispersion profile with (a) $\delta=0.5$ and different values of $\delta_{0}$ or (b) with $\delta_{0}=0.05$, and $\delta=0.125,0.25,1$.

2. Length of DDF $(\mathrm{km})$ as a function of the physical gain $\delta_{0}$, for a virtual gain (a) $\delta=0.5$ and $\underline{\text { CDF length of } 150 \mathrm{~m} \text { (the horizontal arrow indicates a passive DDF) (b) } \delta=0.25 \text {, and CDF }}$

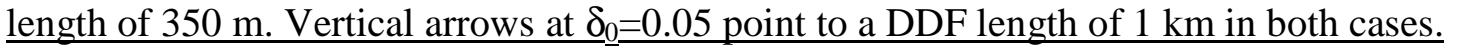

3. Minimum distance for entering the self-similar regime for the equivalent $\mathrm{CDF}$ with $\delta=0.25$, vs. the input parabolic pulse RMS time width, with input pulse energy $\mathrm{U}_{0}=94 \mathrm{pJ}$.

4. Dependence of the ratio $\mathrm{R}$ of gain coefficient-fiber length product $\delta_{0} \mathrm{~L}$ of the DDF and of the equivalent $\mathrm{CDF}$, vs. the DDF gain $\delta_{0}$, for $\delta=0.25$ : the arrow indicates the $\delta_{0}=0.05$ case.

5. (a) Dispersion profile of the DDF for the nonlinear distance $\mathrm{L}_{\mathrm{NL}}=16 \mathrm{~m}(31 \mathrm{~m})$, so that $\delta_{0}=0.025\left(\delta_{0}=0.05\right)$; the virtual $\delta=0.25$ in both cases; (b) DDF length vs. $\delta_{0}$ for $\mathrm{L}_{\mathrm{NL}}=16 \mathrm{~m}$, with $\delta=0.25$ and the CDF length of $175 \mathrm{~m}$. The arrow indicates the $1 \mathrm{~km}$ DDF length for a physical gain of $14 \mathrm{~dB} / \mathrm{km}$.

6. Comparison of the evolution with distance of the RMS time width of a parabolic (Gaussian) pulse in a CDF (dashed (dotted) curve) and in a DDF (solid (dot-dashed) curve) with a physical gain $\delta_{0}=0.1$ in each case, and a virtual gain coefficient $\delta=0.5$ for the DDF.

7. Same as in Fig. 6, for the RMS spectral width.

8. Comparison of the output intensity profile vs. time (top) and frequency (bottom), for a parabolic (Gaussian) pulse after a $1 \mathrm{~km}$ long CDF (grey dashed thick (thin) curves) or DDF (black solid thick (thin) curves) with the physical and virtual gains as in Fig. 6.

9. Evolution of the RMS (a) time and (b) frequency width of a parabolic pulse in a CDF as in Figs. 6-7, but with the linear gain $\delta=0.5$. 
10. Dependence of optimal parabolic pulse parameters vs. its input energy, for a fixed DDF length of $1 \mathrm{~km}$, the physical gain coefficient $\delta_{0}=0.05$, and different virtual gains $\delta$ : (a) optimal input FWHM time width; (b): associated output FWHM spectral width.

11. Same as in Fig. 9, for a DDF gain coefficient $\delta_{0}=0.025$ (with $\mathrm{L}_{\mathrm{NL}}=16 \mathrm{~m}$ ), and $\delta=0.25$.

12. Input (grey dashed curves) and output (solid curves) pulse power profile versus time and frequency for a $1 \mathrm{~km}$ long DDF with (a) $\beta_{3}=0$ with $\delta_{0}=0.1, \delta=0.5$; (b) for a $755 \mathrm{~m}$ long DDF with $\beta_{3}=0.025 \mathrm{ps}^{3} / \mathrm{km}$.

13. Same as Fig. 11(b), for (a) a $779 \mathrm{~m}$ long DDF with $\beta_{3}=0.025 \mathrm{ps}^{3} / \mathrm{km}$; (b) a $974 \mathrm{~m}$ long DDF with $\beta_{3}=0.015 \mathrm{ps}^{3} / \mathrm{km}$. 

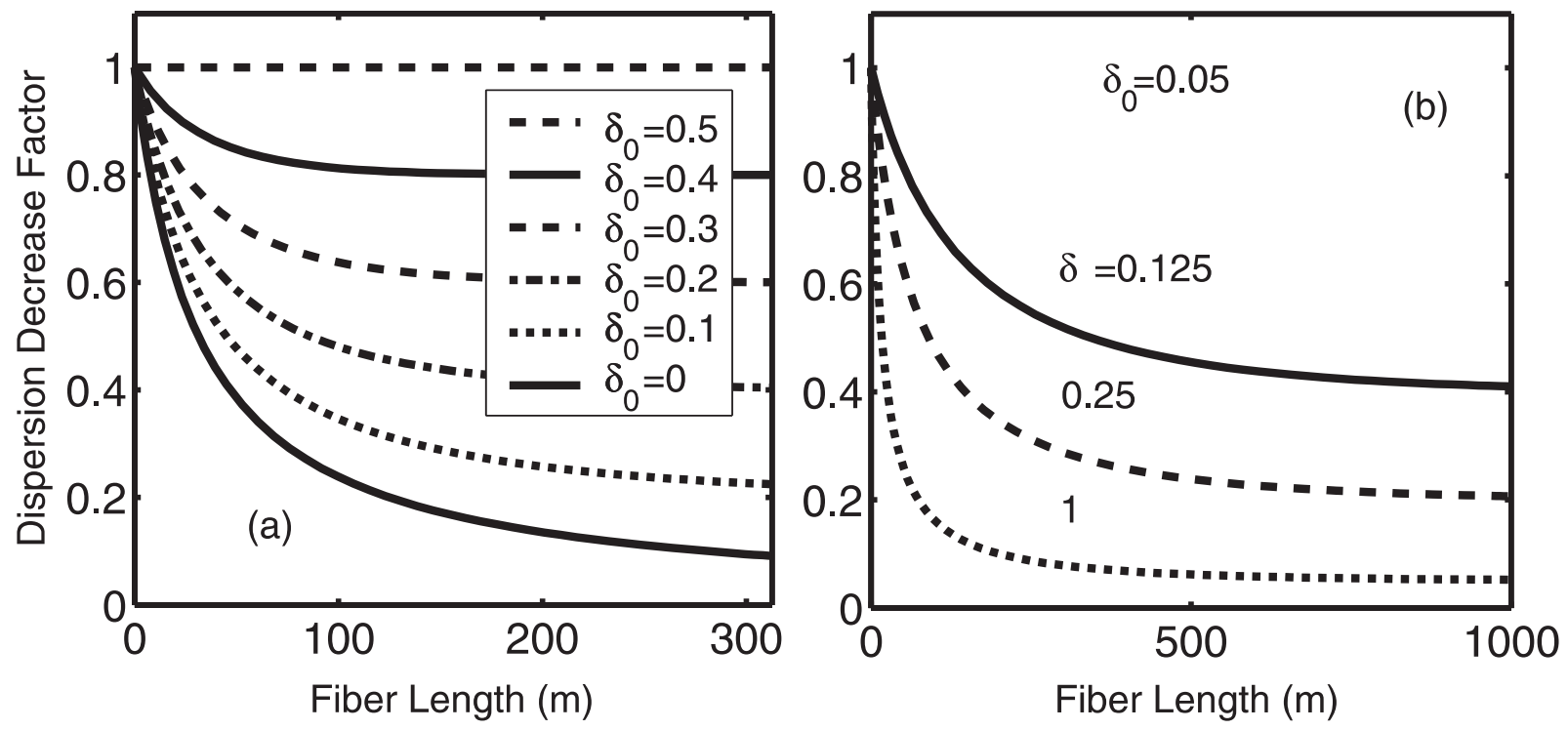

Figure 1
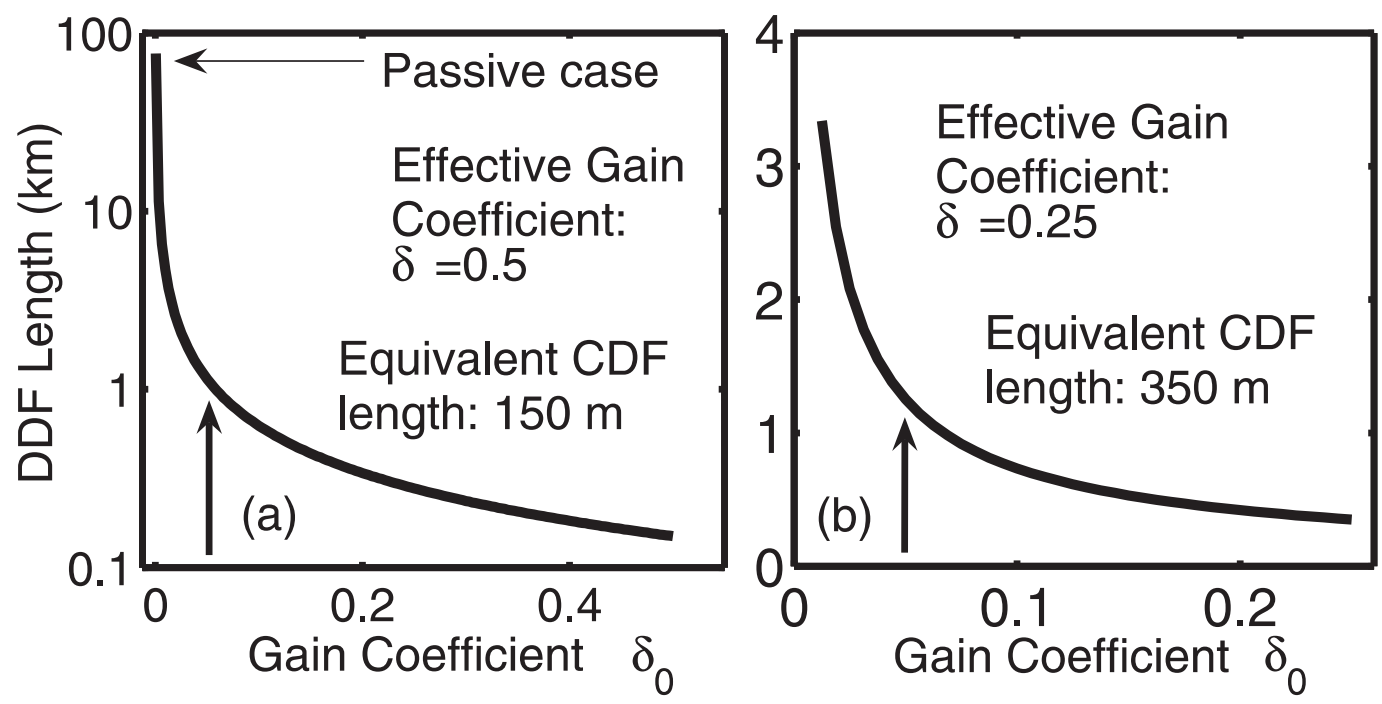

Figure 2 


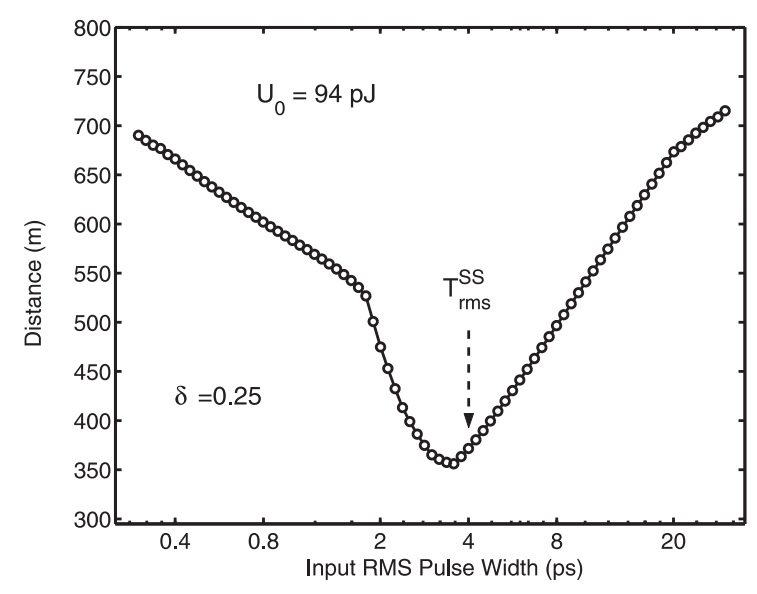

Figure 3

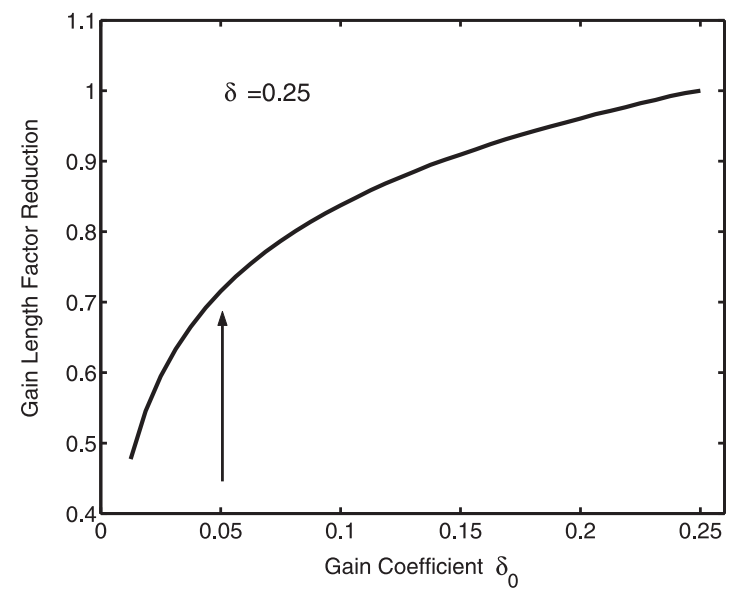

Figure 4
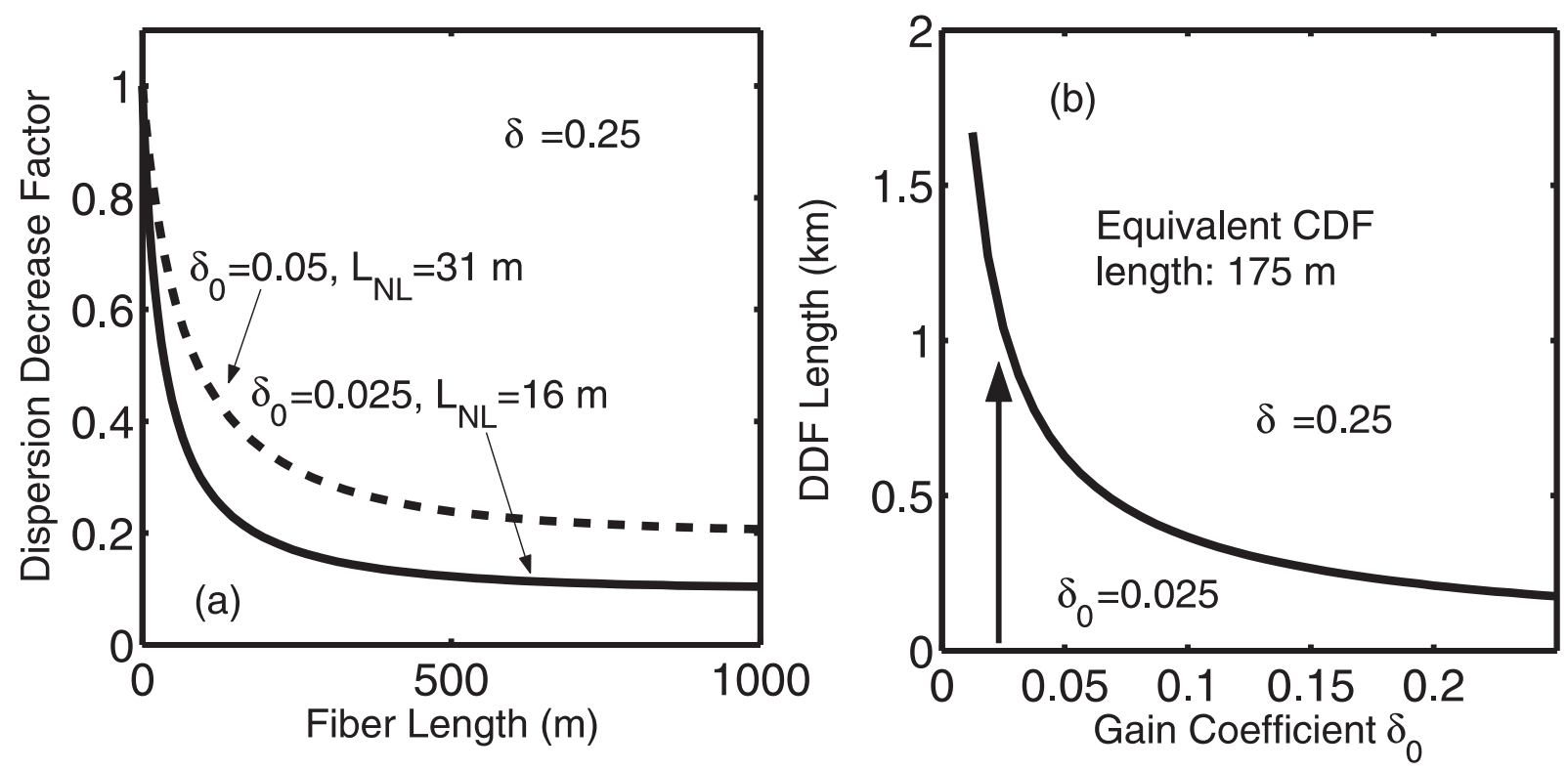

Figure 5 


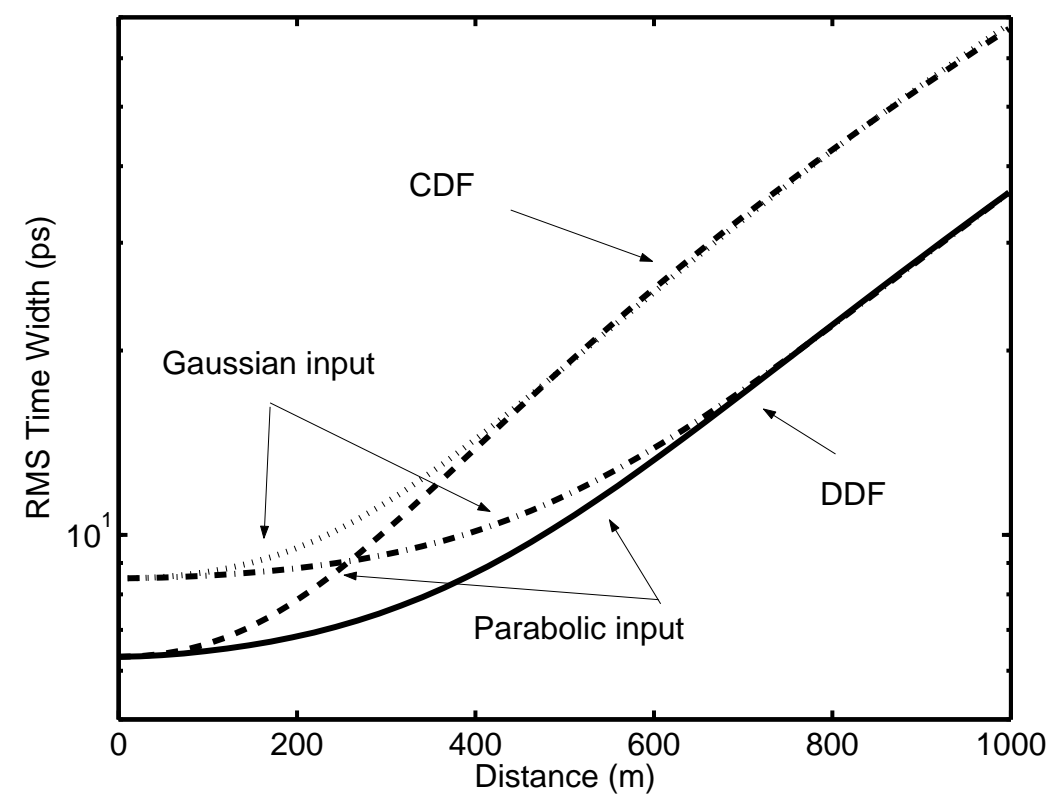

Figure 6

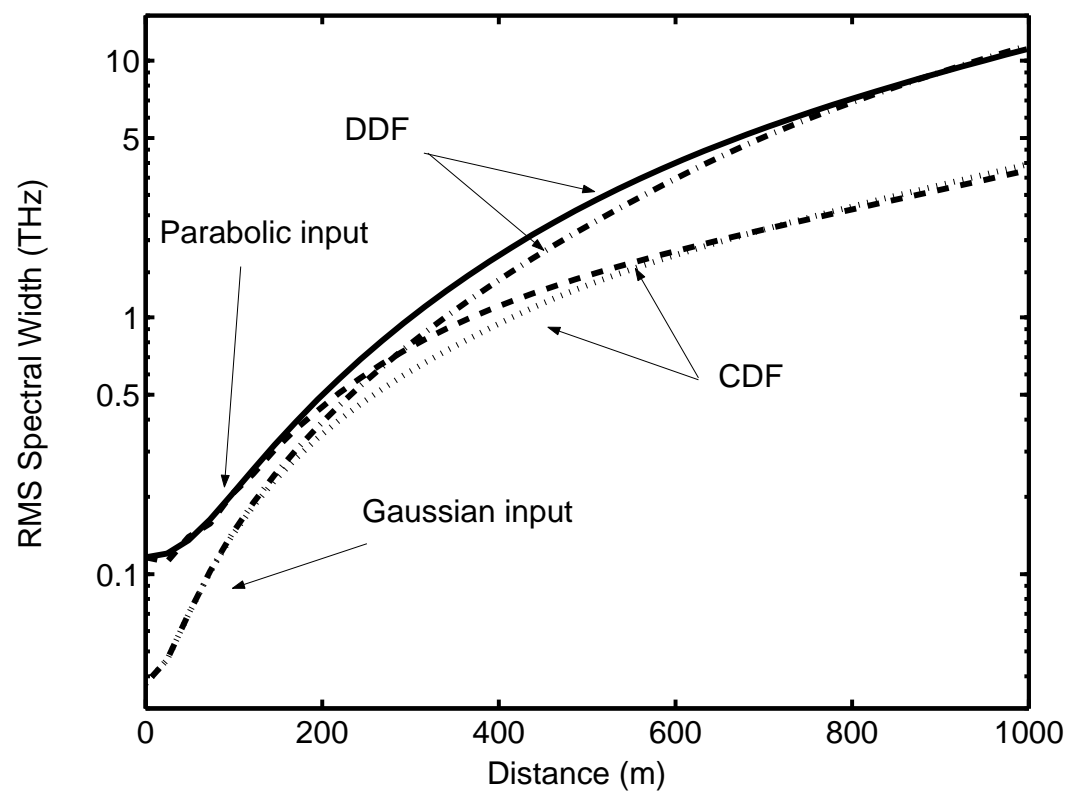

Figure 7 

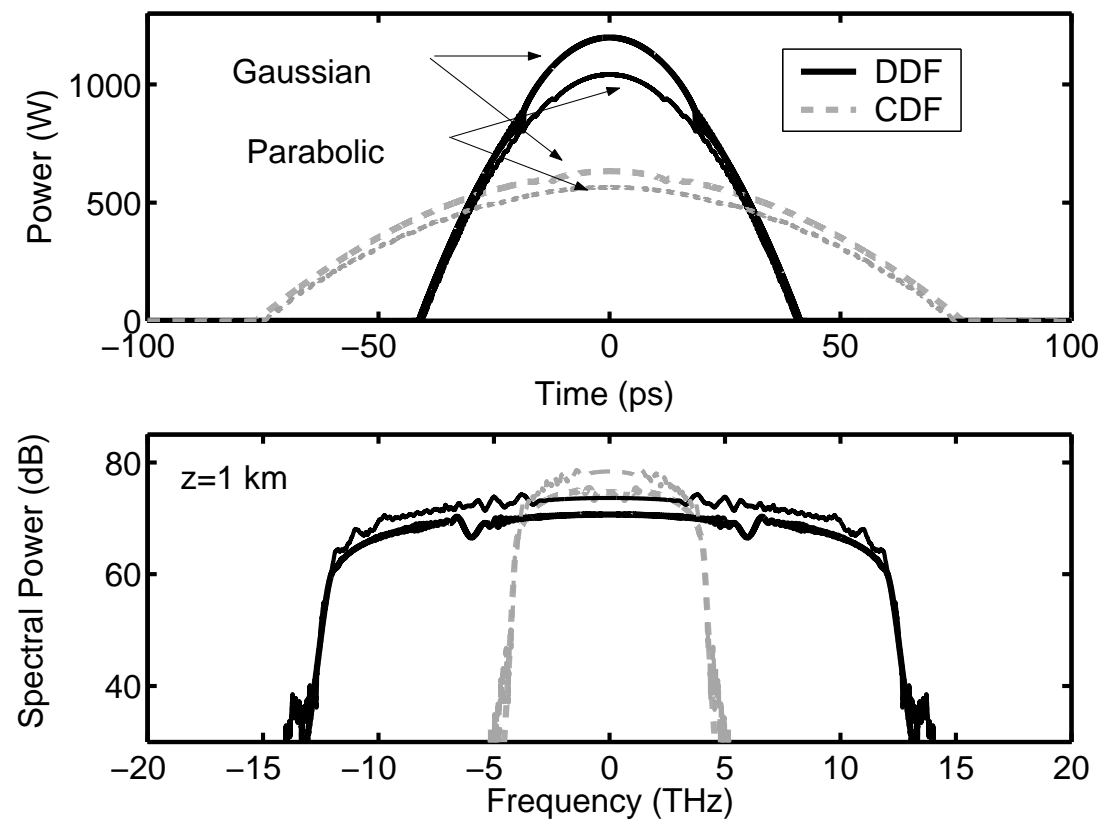

Figure 8
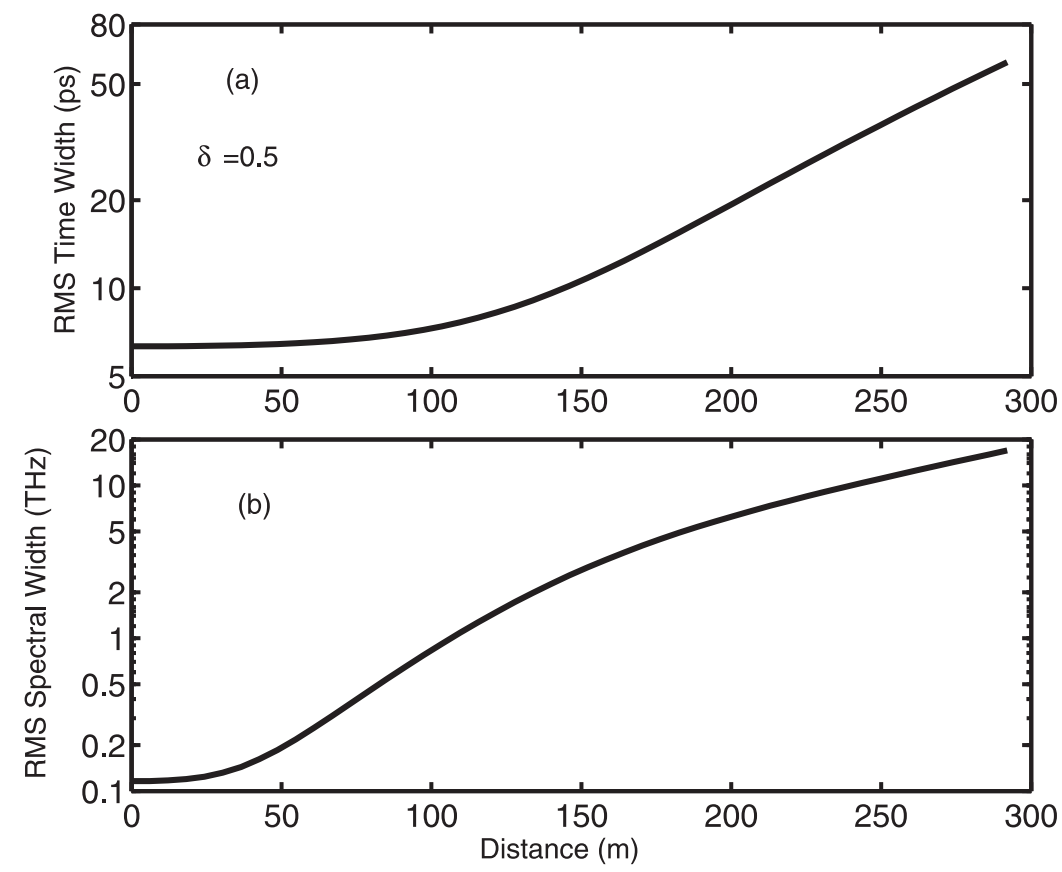

Figure 9 

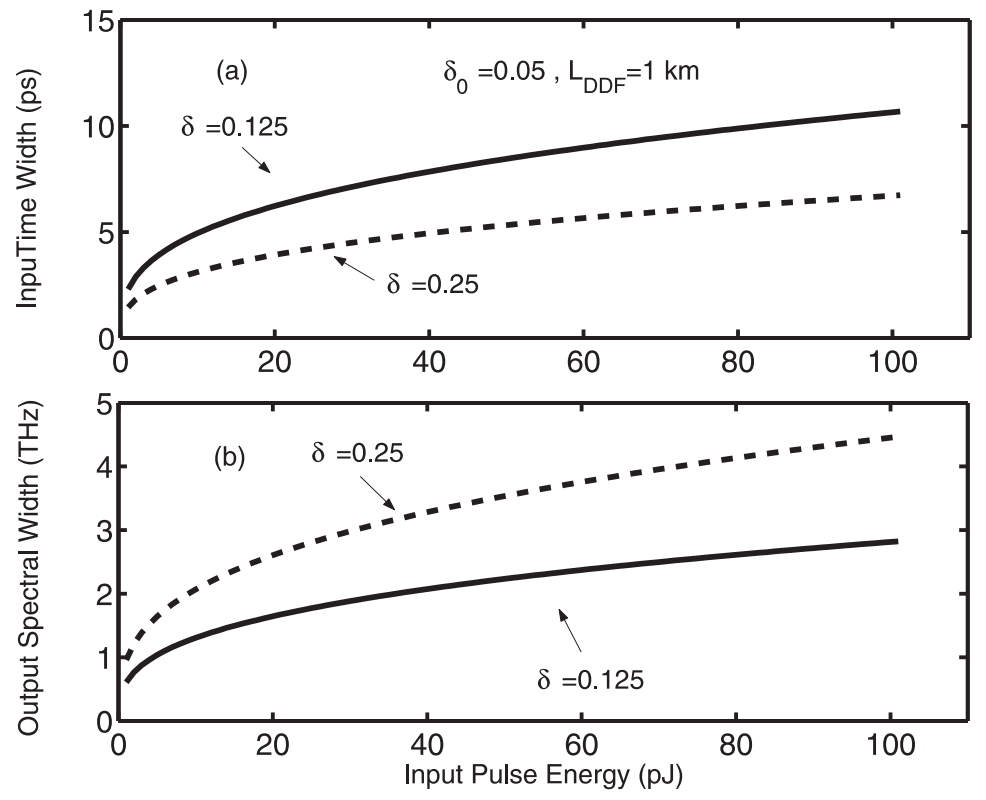

Figure 10
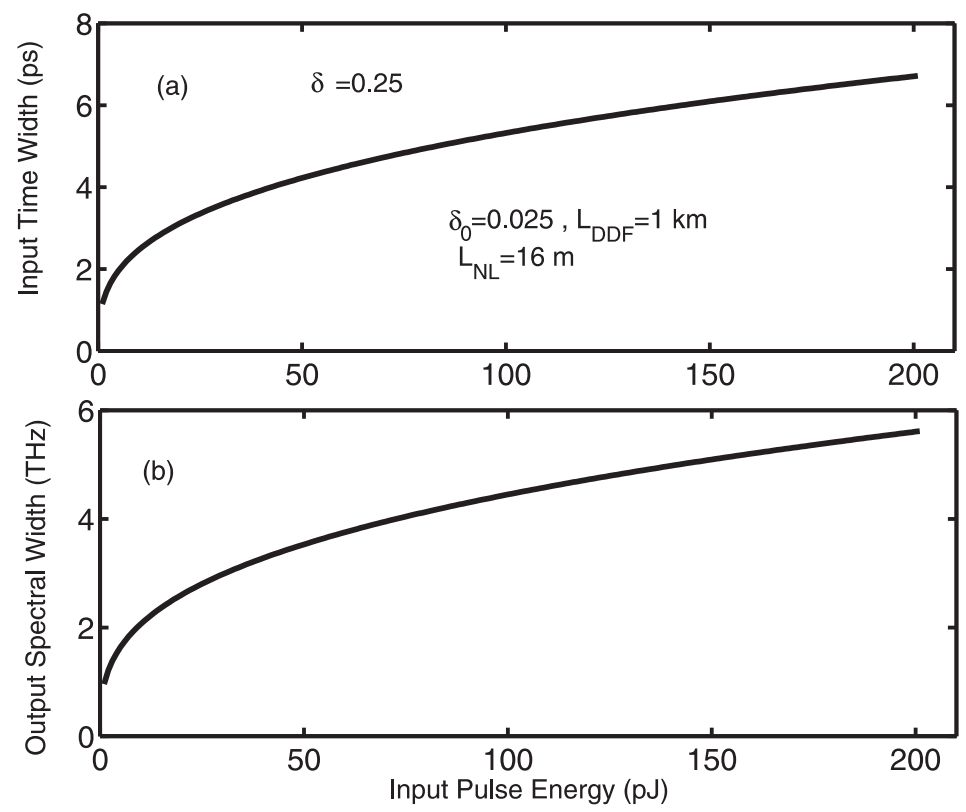

Figure 11 

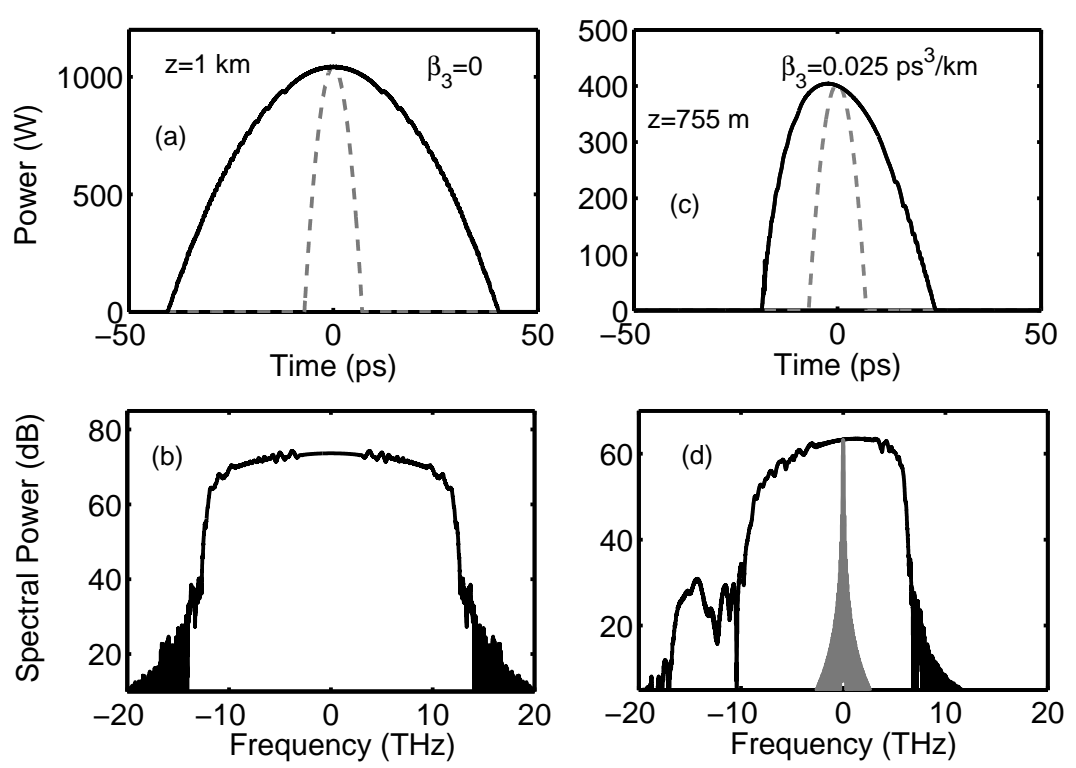

Figure 12
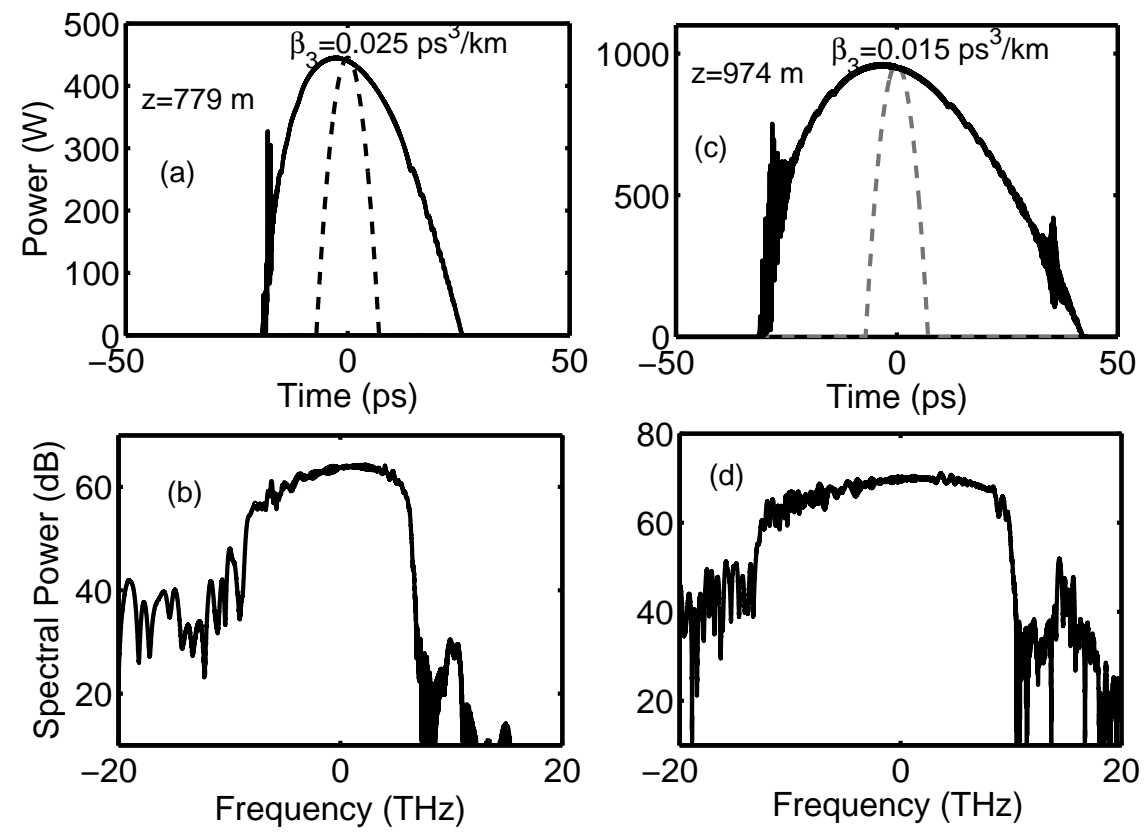

Figure 13 